\section{Effective Integration of Medical and Pharmacy Claims to Better Protect Patient Safety-A Long Road Yet to Travel}

Medical researchers and physicians at the Carolinas Medical Center in Charlotte, NC, reported 3 cases of hepatotoxicity associated with telithromycin (Ketek) in the online edition of Annals of Internal Medicine on January 20, 2006 (in print in the March 21, 2006, issue). ${ }^{1}$ One case involved drug-induced hepatitis that proved to be reversible, 1 patient died from liver failure, and 1 patient required a liver transplant. The drug had been approved by the U.S. Food and Drug Administration (FDA) almost 2 years earlier, on April 1, 2004, and by June 2005, there were 10 cases reported to the FDA of liver problems associated with the use of telithromycin. ${ }^{2}$

The following rates of liver failure per 10 million prescriptions were found by Wall Street Journal researchers for each of the following antibiotics: 23.0 for Ketek, 6.6 for Avelox, 6.0 for Tequin, 4.2 for Biaxin, and 3.7 for Zithromax. ${ }^{2}$ The use of Trovan was sharply restricted in 1999 after the FDA received reports of 14 liver failures, an event rate of 58 per 10 million prescriptions. An FDA memo for incidents reported through April 30, 2006, showed 23 cases of reported serious liver injury and 12 cases of liver failure "associated" with telithromycin. ${ }^{3}$ Telithromycin sales in the United States peaked in 2005 with 3.35 million prescriptions and $\$ 193$ million in manufacturer sales; community pharmacy sales in retail dollars were $\$ 148.3$ million in 2005 , placing telithromycin at rank \#185 among all brand drugs dispensed in the United States. ${ }^{4}$

An FDA physician and reviewer of the Ketek application for approval wrote in April 2007 that there was a serious question about the efficacy of telithromycin since it had been evaluated only in noninferiority trials that had been determined by the FDA to not be "justifiable" for the self-resolving respiratory tract infections for which telithromycin was approved. ${ }^{5}$ In addition, the FDA advisory committee did not see the data integrity violations that were disclosed prior to the meeting of the advisory committee on January 8, 2003, because FDA managers presented study 3014 without mentioning the issues of data integrity. ${ }^{6}$ Among the threats to data integrity that were known at the time were the results of routine FDA investigation of the practices of the physician who enrolled the most patients, more than 400 , in which fraud was uncovered, including complete fabrication of patient enrollment. Not a large wonder since the drug manufacturer paid each physician as much as $\$ 400$ per patient enrolled. More than 24,000 subjects were enrolled from more than 1,800 physicians.

The FDA had relied on study 3104 to defend the safety of telithromycin, but 3 days after a congressional hearing on Ketek in February 2007, the FDA removed any mention of study 3014 from its Web site. Study 3014 was performed by the manufacturer at the request of the FDA following the first round of review of telithromycin in which the advisory committee noted in April 2001 an apparent association with hepatitis, as well as heart and visual side effects, and asked the company to conduct a study that involved patients likely to receive telithromycin if the drug was approved.
The label change for Ketek on February 12, 2007, included the warning about the potential for severe hepatotoxicity, but this warning came 16 months after the first liver failure cases became public. ${ }^{7}$ Equally important, the FDA did not withdraw approval for 2 indications (acute bacterial sinusitis and acute exacerbation of chronic bronchitis), for which the efficacy of Ketek had never been demonstrated, until February 12, 2007. A coincidence perhaps, but the date of this label change was just 1 day before the congressional hearing on Ketek. Ketek is now indicated for the treatment of community-acquired pneumonia (of mild-to-moderate severity) due to Streptococcus pneumoniae (including multi-drugresistant isolates, Haemophilus influenzae, Moraxella catarrhalis, Chlamydophila pneumoniae, or Mycoplasma pneumoniae) for patients aged 18 years and older. The updated label change on February 12,2007 , included a boxed warning and a contraindication stating that no one with myasthenia gravis should take Ketek; warnings were strengthened for loss of consciousness and visual disturbances as well as for the potential for hepatotoxicity.

Using the example of Ketek, Gleason et al. in this issue of $J M C P$ ask the perhaps obvious direct question of what managed care organizations can do to help better protect patient safety and, by implication, what can be done in the face of slow response by the FDA to available evidence of harm. ${ }^{8}$ Among the primary findings of this research were (1) the very small percentage (20 of 507 patients, or 3.9\%) of telithromycin patients with an on-label diagnosis of pneumonia and (2) a prescribing rate that increased only slightly, to 12 of 179 patients (6.7\%) after the formal label change, which narrowed Ketek's indications from 3 to only 1 -communityacquired pneumonia.

Despite the findings of significant off-label prescribing, readers may reasonably question the implication that much of the prescribing of telithromycin was inappropriate. One might even suggest that off-label prescribing of telithromycin was evidencebased. For example, Johnston et al. reported in the New England Journal of Medicine in April 2006 that "this study provides evidence of the benefit of telithromycin in patients with acute exacerbations of asthma." Probing this research shows that telithromycin had no effect on peak expiratory flow in the morning in these adult asthma patients but was associated with a statistically significant reduction in asthma symptoms compared with placebo. However, the practical clinical effect of this statistically significant outcome is questionable. On a 7-point scale, with 0 for no symptoms and 6 for severe symptoms, the average difference in the decrease in asthma symptom scores was - 0.3 (1.3 for telithromycin vs. 1.0 for placebo), 95\% confidence interval -0.5 to $-0.1, P=0.004$. Probing further, the average self-reported asthma symptom score decreased in the placebo group from 2.8 to 1.8 and in the telithromycin group from 3.0 to 1.7. Some New England Journal of Medicine readers may have seen the accompanying editorial by Little that cautioned against using the results of the TELICAST (Telithromycin, Chlamydophila, and Asthma) study to justify telithromycin to treat acute exacerbations of asthma. ${ }^{10}$ 
In hindsight, the potential risks outweigh the benefits for most patients who received telithromycin, and the conclusions of The Medical Letter consultants in their review in August 2004 did not dissuade prescribing of telithromycin: while expensive, telithromycin "would be a reasonable alternative to a fluoroquinolone for the treatment of such infections" (bacterial respiratory infections and pneumococcal respiratory infections). ${ }^{11}$ Yet, telithromycin was promoted by its manufacturer for the first-line treatment of everyday respiratory infections during a period in which worldwide attention was being focused on the contribution of overuse of antibiotics to bacterial resistance. ${ }^{12}$

The data presented by Gleason et al. may therefore represent an indictment of physician prescribing of telithromycin and prescriber susceptibility to overpromotion of the drug. But interpretation of the data presented by Gleason et al. may be informed by 4 additional points. First, the starting day of the period for data capture after the label change was the very next day, February 13, 2007. It is not clear from the data presented by Gleason et al. how many of the 179 patients prescribed telithromycin between February 13, 2007, and April 13, 2007, had a medical diagnosis prior to the date of the label change, presumably as early as January 11, 2007. Second, the researchers may have refined their analysis by examining the date-written field in the pharmacy claims in addition to the date filled. It is possible, and even likely, that some of these telithromycin claims were associated with prescriptions that had been written weeks prior to the label change on February 12, 2007. Third, the diagnoses recorded in administrative claims have been shown to be unreliable. ${ }^{13}$ Fourth, approximately 34\% of the telithromycin patients before and after the label change had a diagnosis of sinusitis, and the clinical differentiation of viral from bacterial sinusitis is difficult in the absence of a bacteriologic culture. ${ }^{14}$

However, these points do not diminish the concern raised by the data presented by Gleason et al. that almost half of the patients who received telithromycin had either apparently minor upper respiratory tract infections, an indication that this drug does not address well if at all, or a medical diagnosis not related to FDAapproved indications. These patients were exposed to avoidable risk. The first cut of the claims data performed by Gleason et al. showed that 75 of 507 patients (14.8\%) prescribed telithromycin had a medical claim for unspecified upper respiratory infection, which is typically viral in nature.

One final point in the data presented should give us further pause: approximately $14 \%$ of the patients with a telithromycin claim had no associated medical claim from which to determine diagnosis. In other words, when put to this test, the integration of pharmacy and medical claims still leaves us short of the ability to adequately mine administrative claims data. We need better mechanisms to promote patient safety and monitor the indications for which medications are prescribed.

Frederic R. Curtiss, PhD, RPh, CEBS Editor-in-Chief fcurtiss@amcp.org

\section{REFERENCES}

1. Clay KD, Hanson JS, Pope SD, Rissmiller RW, Purdum PP, Banks PM. Brief communication: severe hepatotoxicity of telithromycin: three case reports and literature review. Ann Intern Med. 2006;144:415-20.

2. Dooren JC. Sanofi antibiotic may be tied to liver problems, doctors say. Wall Street Journal. January 21-22, 2006:A6.

3. Mathews AW. FDA panel urges prominent warning label for antibiotic. Wall Street Journal. May 19, 2006:B1, B2

4. Community practice. Top 200 brand-name drugs by retail dollars in 2005 Drug Top. March 6, 2006:26.

5. Ross DB. The FDA and the case of Ketek (commentary). N Engl J Med. 2007;356:1601-04.

6. Letter from Senator Charles Grassley to FDA Commissioner Andrew C von Eschenbach, December 13, 2006. Available at: http://finance.senate.gov/press/ Gpress/2005/prg121306a.pdf. Accessed May 30, 2007.

7. U.S. Food and Drug Administration, Center for Drug Evaluation and Research. Telithromycin (marketed as Ketek) information. February 12, 2007. Available at: http://www.fda.gov/cder/drug/infopage/telithromycin/default.htm. Accessed May 30, 2007

8. Gleason PP, Walters C, Heaton AH, Schafer JA. Telithromycin: the perils of hasty adoption and persistence of off-label prescribing. J Manag Care Pharm. 2007;13(5):420-25. Available at: http://www.amcp.org/data/jmcp/420-25.pdf.

9. Johnston SL, Blasi F, Black PN, Martin RJ, Farrell DJ, Nierman RB, for the TELICAST Investigators. The effect of telithromycin in acute exacerbations of asthma. N Engl J Med. 2007;354:1589-1600.

10. Little FF. Treating acute asthma with antibiotics—not quite yet, N Engl J Med. 2007;354:1632-34.

11. Telithromycin (Ketek) for respiratory infections. Med Letter. August 16, 2004;46:66-68.

12. Mintzes B. Pharmaceutical promotion and prevention of antibiotic resistance [abstract]. October 2005. Available at: http://www.pasteur.fr/applications/ euroconf/antiinfectionstherapies/7_Mintzes_abstract_pdf. Accessed June 2, 2007.

13. Peabody JW, Luck J, Jain S, Bertenthal D, Glassman P. Assessing the accuracy of administrative data in health information systems. Med Care. 2004; 42(11):1066-72.

14. Chadha NK, Chadha R. 10-minute consultation: sinusitis. BMJ. 2007;334: 1165. [doi:10.1136/bmj.39161.557211.47].

\section{Letters to the Editor}

JMCP welcomes letters that serve to clarify subjects published in previous issues of the Journal or regarding subject matter of interest to managed care pharmacists. Letters in JMCP are not peer reviewed but are subjected to editorial review. Letters should be prepared in a word processing program, preferably Microsoft Word, and submitted electronically at jmcp.msubmit.net. See www.amcp.org for details. 